Short communication

Agronomy

\title{
Germination sexuée et croissance précoce d'Ozoroa insignis Del., une espèce médicinale du Burkina Faso
}

\author{
Patrice ZERBO1.3*, Bassirou BELEM², Jeanne MLLOGO-RASOLODIMBY¹ \& Patrick VAN DAMME ${ }^{3}$
}

1. Laboratoire de Biologie et Ecologie Végétales (La.B.E.V.), UFR Sciences de la Vie \& Terre, 09 BP 848, Université de Ouagadougou, Burkina Faso.

2. Centre National de Semences Forestières, 01 BP2682 Ouagadougou 01, Burkina Faso

3. Laboratoire d'Agronomie Tropicale et Subtropicale et d'Ethnobotanique, Faculté des Sciences en Bio-ingénierie, Coupure links 653, 9000 Gent, Université de Gand, Belgique.

*. Auteur correspondant : patrice zerbo@univ-ouaga.bf / patrice.zerbo@ugent.be

\section{RESUME}

L'engouement pour l'utilisation des espèces médicinales constitue une menace sérieuse pour certaines espèces au Burkina Faso. Des solutions impératives devraient être trouvées en vue d'une gestion durable desdites espèces. Dans cette optique, une étude sur la germination sexuée d'Ozoroa insignis Del. a été conduite au laboratoire et en pépinière. L'effet du prétraitement et celui substrat sur la germination des semences ont été étudiés. Le développement morphologique précoce des plantules a été aussi étudié. Les résultats ont révélé que la germination est influencée par le type traitement et du substrat utilisé. Le traitement acide et le substrat sableux offrent de meilleurs taux moyens de germination. Les fortes chaleurs (cuisson et ébouillantage) semblent néfastes aux semences. Les plantules ont présenté un développement précoce. Cette croissance rapide est un atout à la régénération en milieu naturel.

Mots-clés : Ozoroa insignis, Germination sexuée, Croissance, Burkina Faso

\section{ABSTRACT}

The great medicinal plant used in Burkina Faso is a serious threat of some of them. Compelling solutions should be found for these species sustainable management. Then, Ozoroa insignis Del sexual germination were conducted in the laboratory and nursery. The effect of pretreatment and the substrate on seed germination were studied. Seedlings morphological growing was also studied. The results showed that germination is influenced by the treatment and the substrate used. The acid treatment and the sandy substrate offer better means of germination. High temperature (cooking and boiling) seem harmful to seeds. Seedlings showed a quite development taproot system. This rapid growth is an asset for regeneration in the wild.

Keywords: Ozoroa insignis, Sexual germination, Growth, Burkina Faso

\section{INTRODUCTION}

Au Burkina Faso comme dans d'autres pays sahéliens, les formations végétales rencontrées, sont les savanes arbustives ou arborées, les steppes arbustives et quelques forêts claires. Ces formations végétales jouent un grand rôle dans la sécurisation des conditions de vie des populations en leur procurant aliments, produits médicinaux, matériaux de construction, fourrage et divers autres services (ombrage, fertilisation des sols, agrémentation du milieu). Si les utilisations alimentaires sont répandues, celles liées à la médecine traditionnelle sont aussi importantes. En effet, la majeure partie de la population du Burkina Faso (90 \%) a tendance à se soigner elle-même en utilisant des produits de la pharmacopée traditionnelle préparés à base de plantes [1]. Plusieurs plantes médicinales ont ainsi fait l'objet d'un recensement exhaustif et de la détermination des vertus curatives [2 - 5]. Outre ces utilisations locales, les exportations annuelles des dites plantes atteindraient environ deux millions de tonnes générant ainsi des recettes de plus de dix milliards de francs CFA [6]. Malheureusement, cet engouement pour les plantes, associé au manque de textes officiels règlementant la collecte et la commercialisation des plantes médicinales a engendré un type nouveau de pression sur la végétation. A la perte de la diversité biologique que va engendrer une telle menace, s'ajoute une perte des habitats naturels des espèces $[3,7]$. Dans l'optique d'une gestion durable de ces plantes médicinales, la régénération des espèces médicinales s'avère donc une nécessité. Leur mise en culture et leur domestication dans les bosquets ou les champs permettraient ainsi de 
suppléer à la régénération naturelle fréquemment compromise par les pressions humaines et animales et, de contribuer à la promotion de ces espèces [8].

De nombreux travaux de recherche ont déjà abordé la problématique de la régénération assistée des espèces végétales ligneuses en Afrique tropicale sèche $[9,10]$. Au Burkina Faso, plusieurs espèces ont fait l'objet de travaux: Combretum micranthum [11]; Boswellia dalzielii [12]; Piliostigma reticulatum [13]; Bombax costatum [14]; etc. Cependant, peu de documents portent sur la la régénération assistée des plantes médicinales surtout, celles à large spectre thérapeutique dont Ozoroa insignis Del. (Anacardiaceae). Cette plante est largement utilisée par les populations locales africaines pour les soins de santé. Ses feuilles sont utilisées contre la diarrhée, les coliques [4] au Burkina Faso tandis que les racines sont utilisées dans les traitements de la syphilis et l'asthénie [2]. Au Bénin, l'espèce serait utilisée comme un agent galactogène et antiépileptique [15] tandis qu'au Zimbabwé, l'infusion des racines ou des écorces est utilisée dans les soins de la diarrhée et les maladies vénériennes [16]. L'activité antiparasitaire de la plante est exploitée dans le traitement du paludisme au Ghana [17]. En Tanzanie comme en Uganda, l'espèce est utilisée dans les traitements des infections à candida [18]. Comme pour la plupart des plantes médicinales, sa culture en pépinière suivi de plantation est inexistante. En raison de sa forte valeur thérapeutique, la nécessité de proposer une solution de gestion durable est indéniable. La présente étude dont l'objectif est d'évaluer l'aptitude de la production de plants en pots des semences d'O. insignis répond à ce souci. II s'est agi de déterminer les prétraitements appropriés et les substrats les mieux adaptés pour faire germer les semences. En outre, la dynamique de la croissance morphologique de l'espèce a été également étudiée.

\section{MATERIEL ET METHODES Collecte des données}

Le matériel était constitué des semences d'O. insignis collectées sur des semenciers localisés dans le secteur phytogéographique nordsoudanien au Burkina Faso [19]. La semence est une drupe noire aplatie verticalement et dure mesurant $12 \mathrm{~mm}$ de long [20]. Les semences récoltées ont été conservées au laboratoire, en température ambiante $\left(25-30^{\circ} \mathrm{C}\right)$ pendant un mois. Au moment des semis, ces semences avaient une teneur en eau de 7,96 \%.

Avant les semis, 14 types de prétraitements (Tableau 1) ont été appliqués aux semences, suivant les normes 2009 de l'ISTA (International Seed Testing Association). Ainsi, deux groupes de semences ont été mises à germer : les semences non traitées (témoins) et les semences traitées soit à l'eau, à la chaleur (cuisson ; ébouillantage), à l'acide ou scarifiées.

Deux types de substrats ont été utilisés au cours de ces essais de germination :

- un substrat composé du sable fin de rivière ou substrat SAR, mis dans de petites boîtes à germination en plastique (Planche 1). Pour chaque traitement, quatre répétitions de 50 semences soit dix par boîtes de germination ont été effectuées. Après les semis, les boîtes ont été déposées au laboratoire et arrosées régulièrement.

- un substrat composé d'un mélange de sable (1/3), du fumier (1/3) et du sol (2/3) ou substrat (SFS). Des pots en sachets plastiques noirs opaques et perforés ont été remplis par ce substrat. Pour chaque traitement, le dispositif expérimental était du type blocs complètement randomisés avec quatre blocs. Le bloc comprenait 25 pots disposés en carré à raison de cinq (5) pots par côté (Planche 1). Au total 100 semences ont été utilisés pour chaque traitement soit une semence par pot. Tous les pots ont été ensuite déposés à la pépinière et arrosés régulièrement.

Les semences émergées du substrat ont été comptées comme germées et inscrites sur des fiches de germination. Le comptage s'est effectué tous les trois jours jusqu'au 30è jour de germination, période au-delà de laquelle aucune semence n'avait germé.

Le développement morphologique des plantules a été estimé par l'étude de la croissance de la tigelle. Chaque semaine, les hauteurs de cinq des tigelles sont mesurées et inscrites sur des fiches de croissance. Les mesures se sont déroulées durant dix semaines (70 jours) car à partir de 2 à 4 mois, les espèces ligneuses peuvent être transplantées [20]. 
Tableau1: Différents types de prétraitements appliqués aux semences

\begin{tabular}{|c|c|}
\hline Codes & Prétraitements \\
\hline $\mathrm{T}_{0}$ & Témoin = Sans prétraitement \\
\hline $\mathrm{T}_{1}$ & Trempage à l'eau durant 24 heures \\
\hline $\mathrm{T}_{2}$ & Trempage à l'eau durant 48 heures \\
\hline $\mathrm{T}_{3}$ & $\begin{array}{l}\text { Ebouillantage suivi d'un trempage à l'eau } \\
\text { durant } 24 \text { heures }\end{array}$ \\
\hline $\mathrm{T}_{4}$ & $\begin{array}{l}\text { Ebouillantage suivi d'un trempage à l'eau } \\
\text { durant } 48 \text { heures }\end{array}$ \\
\hline$T_{5}$ & $\begin{array}{l}\text { Cuisson durant } 1 \text { minute suivie d'un } \\
\text { trempage à l'eau pendant } 24 \text { heures }\end{array}$ \\
\hline $\mathrm{T}_{6}$ & $\begin{array}{l}\text { Cuisson durant } 5 \text { minutes suivie d'un } \\
\text { trempage à l'eau pendant } 24 \text { heures }\end{array}$ \\
\hline $\mathrm{T}_{7}$ & $\begin{array}{l}\text { Cuisson durant } 10 \text { minutes suivie d'un } \\
\text { trempage à l'eau pendant } 24 \text { heures }\end{array}$ \\
\hline $\mathrm{T}_{8}$ & $\begin{array}{l}\text { Trempage à l'acide sulfurique }\left(\mathrm{H}_{2} \mathrm{SO}_{4}\right) \text { à } \\
98 \% \text { suivi durant } 1 \text { minute d'un trempage } \\
\text { à l'eau pendant } 24 \text { heures }\end{array}$ \\
\hline $\mathrm{T}_{9}$ & $\begin{array}{l}\text { Trempage à l'acide sulfurique }\left(\mathrm{H}_{2} \mathrm{SO}_{4}\right) \text { à } \\
98 \% \text { durant } 5 \text { minutes suivi d'un } \\
\text { trempage à l'eau pendant } 24 \text { heures }\end{array}$ \\
\hline $\mathrm{T}_{10}$ & $\begin{array}{l}\text { Trempage à l'acide sulfurique }\left(\mathrm{H}_{2} \mathrm{SO}_{4}\right) \text { à } \\
98 \% \text { durant } 10 \text { minutes suivi d'un } \\
\text { trempage à l'eau pendant } 24 \text { heures }\end{array}$ \\
\hline $\mathrm{T}_{11}$ & $\begin{array}{l}\text { Trempage à l'acide sulfurique }\left(\mathrm{H}_{2} \mathrm{SO}_{4}\right) \text { à } \\
98 \% \text { durant } 30 \text { minutes suivi d'un } \\
\text { trempage à l'eau pendant } 24 \text { heures }\end{array}$ \\
\hline $\mathrm{T}_{12}$ & $\begin{array}{l}\text { Trempage à l'acide sulfurique }\left(\mathrm{H}_{2} \mathrm{SO}_{4}\right) \text { à } \\
98 \% \text { durant } 60 \text { minutes suivi d'un } \\
\text { trempage à l'eau pendant } 24 \text { heures }\end{array}$ \\
\hline $\mathrm{T}_{13}$ & $\begin{array}{l}\text { Scarification suivie d'un trempage dans } \\
\text { l'eau pendant } 24 \text { heures }\end{array}$ \\
\hline
\end{tabular}

Légende : $\mathrm{T}$ : traitement
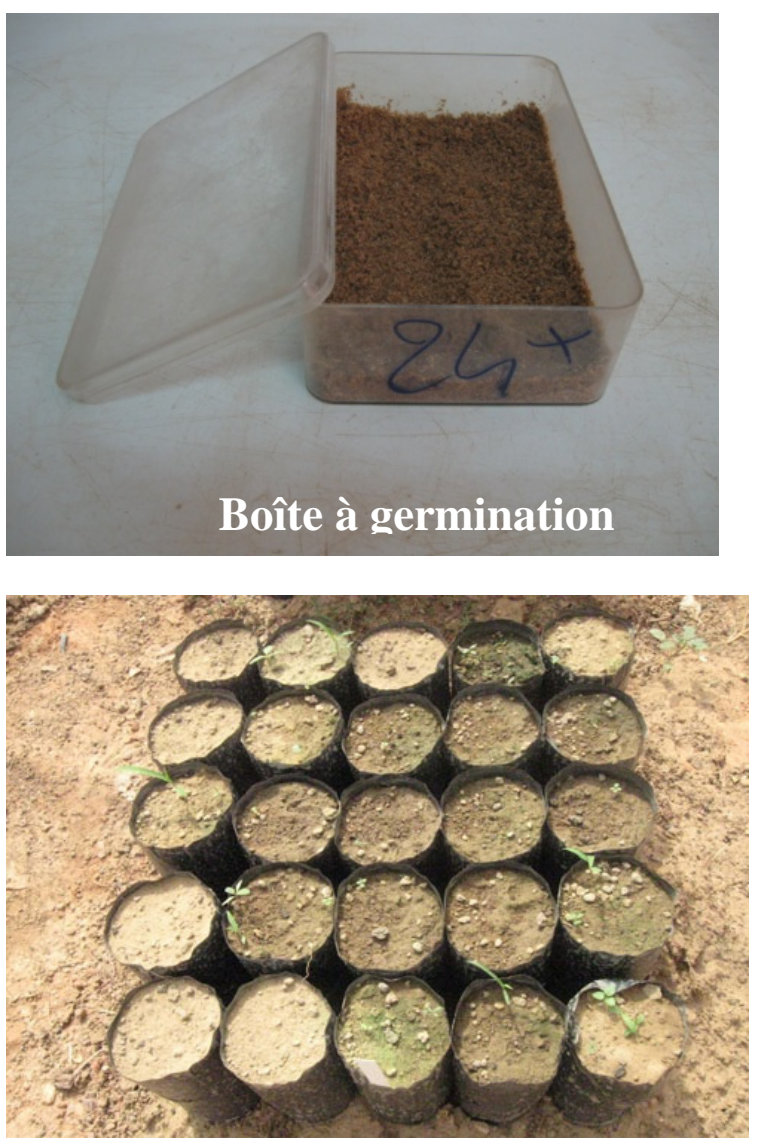

Planche 1 : Supports de germination

\section{Analyse des données}

Le taux moyen de germination (TMG) a été calculé en fonction du type de prétraitement et du substrat selon la formule suivante:

$T M G(\%)=\frac{n}{N} \times 100$

avec $n$, le nombre de semences germées et $N$, nombre de semences semées.

Les TMG des semences prétraitées ont été comparés avec ceux des semences témoins. Ces données ont été ensuite analysées à l'aide du logiciel SAS 6 (Statistical Analysis System). Nous avons procédé à l'analyse de variances à deux facteurs : le traitement et le substrat. Les facteurs ont été croisés, et à chaque fois qu'un facteur s'est montré significatif, nous avons procédé à une comparaison de moyennes par le test de Student-Newman-Keuls pour la variable au seuil de signification de $5 \%$. En outre, les effets du prétraitement et ceux du substrat sur la germination ont été évaluées. 
Au niveau de la croissance, les hauteurs moyennes (HM) des plantules ont été calculées durant dix semaines. La croissance précoce a été estimée.

\section{RESULTATS}

\section{Essais de germination}

L'interaction entre le traitement appliqué et le substrat $(\mathrm{Pr}<0,001)$ influence significativement la germination. Les trempages dans l'acide $\left(T_{8}, T_{9}\right.$ $\mathrm{T}_{10}, \mathrm{~T}_{11}, \mathrm{~T}_{12}$ ) et le substrat SAR offrent des meilleurs taux moyens de germination (TMG) par rapport aux autres traitements (Figure 1): le maximum de germination (74 \%) est obtenu avec le trempage dans l'acide tandis qu'il est de $30 \%$ sur le SFS. En outre, le temps de latence est de six jours sur le SAR et, neuf jours sur le SFS. Quant à l'ébouillantage $\left(T_{3}, T_{4}\right)$ et à la cuisson $\left(T_{5}, T_{6}, T_{7}\right)$, elles semblent défavorables à germination, aucune semence (0\%) n'ayant germé.

\section{Dynamique de la croissance des plantules}

La croissance observée est du type épigé. L'élongation de l'hypocotyle entraine les cotylédons hors du sol. Initialement courbée en crosse, l'hypocotyle se redresse par la suite. Simultanément, la gemmule et les cotylédons sont exposés. Sur les deux types de substrats, deux phases ont été observées dans le développement morphologique (Figure 2) : une phase de croissance rapide pendant les six premières semaines (S1 à $\mathrm{S} 6$ ) et une phase de ralentissement de la croissance de la semaine 7 (S7) à S10. Cependant, la croissance caulinaire est meilleure sur le substrat SAR.

\section{DISCUSSION ET CONCLUSION}

L'étude de la germination des semences d'O. insignis Del. a révélé des effets significatifs des prétraitements et du substrat sur le TMG. Les meilleurs TMG ont été obtenus avec le traitement acide. Ces résultats pourraient s'expliquer par la structure externe de la semence. En effet, celle-ci est recouverte d'une forte couche grasse. Le trempage dans l'acide aurait donc permis d'ôter cet ectocarpe gras favorisant la germination rapide de l'embryon. Pourtant les traitements thermiques, (ébouillante et la cuisson) ont semblés être néfastes à la germination car aucune semence n'a germé. L'absence de germination des semences serait probablement due à la mort du germe de l'embryon. En effet, la semence en général et l'embryon qu'elle contient en particulier, sont très sensibles à de hautes températures. Des résultats semblables ont été obtenus au cours des essais de germination respectivement réalisés sur Securidaca longepedunculata Fresen. [21] et Ximenia americana L. [22].

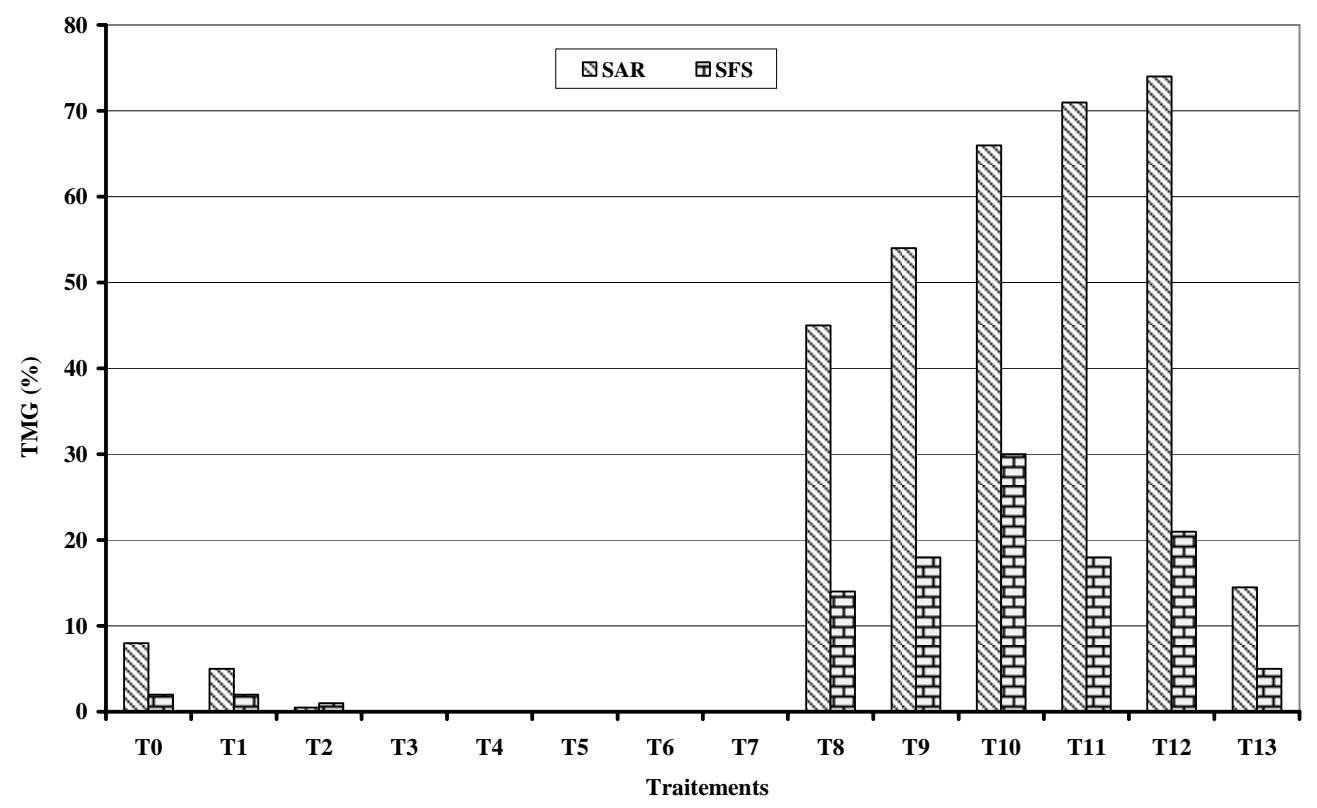

Figure 1: Effets du traitement sur la germination 


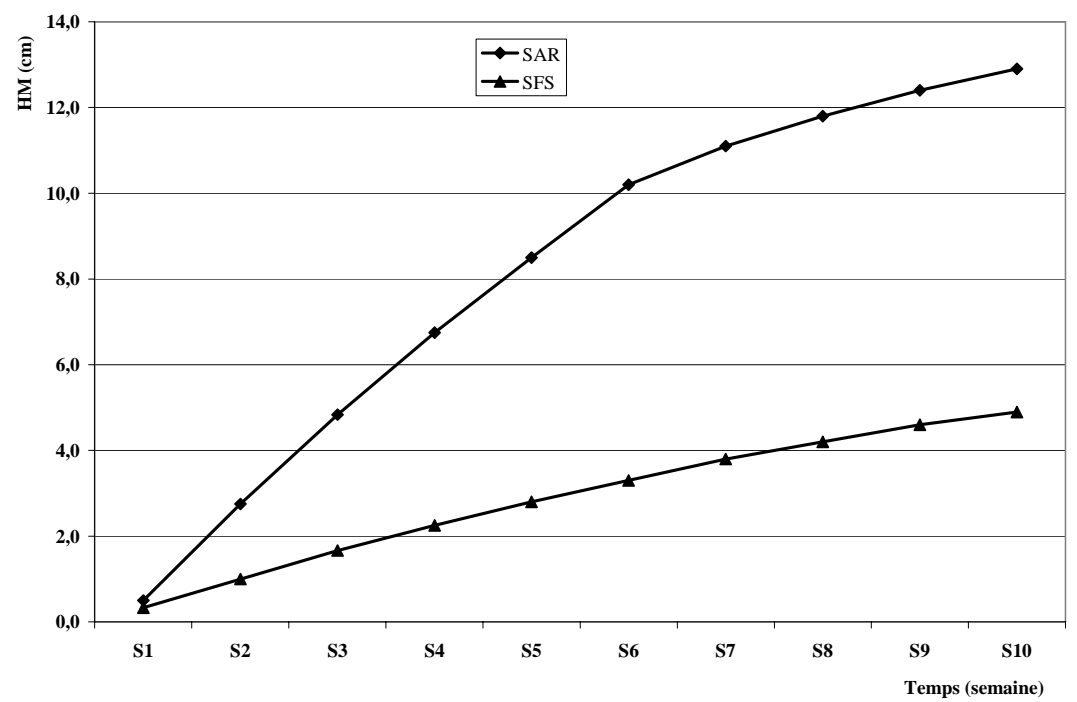

Figure 2 : Elongation moyenne de la tige des plantules

Au niveau du substrat, les meilleurs TMG ont été observés sur le substrat sableux (74\%). Sa bonne infiltration de l'eau et son aération, comparativement au substrat mélangé (SFS) sont à la base de ces meilleurs TMG. En effet, un embryon est mal approvisionné en oxygène ne germe pas. En outre, les semences récalcitrantes ont une faible longévité [23]. Pourtant, les faibles TMG enregistrés au niveau du substrat SFS pourraient être imputables aux contaminations des parasites contenus dans le fumier utilisé. Ces parasites attaquent les germes provoquant des fontes de pré-émergence [24]. L'impact du substrat sur le taux de germination a été observé au cours des conditions de germination de quatre espèces de Combretaceae (Combretum aeculeatum, C. glutinosum, C. micranthum, C. nigricans) [11] ou la germination de Boswelia dalzeilii [12] ou celle de Piliostigma reticulatum [13]. Pour ces auteurs, la structure et la texture du substrat influencent la germination car elles déterminent sa porosité, par conséquent une bonne aération et une pénétration de l'eau.

Au niveau du développement morphologique, une croissance précoce a été observée chez toutes les plantules. Pendant les six semaines suivant la germination, la croissance est rapide ; elle ralentit par la suite entre 7è et la 10è semaine. Des résultats similaires ont été obtenus au cours des essais de germination de Piliostigma reticulatum [13]. La croissance précoce serait due à activité intense des méristèmes, tissus embryonnaires responsables de la croissance logés dans la zone subapicale des tiges et des racines. Les cotylédons toujours attachés à la plante, assurent la nutrition équilibrée des plantules. Leur chute correspond à la période de ralentissement de la croissance, qui marque le début de l'autotrophie des plantules. les plantules doivent alors rechercher la matière minérale pour synthétiser elles-mêmes sa matière organique. A cette période, le bourgeon apical est devenu moins actif d'où le ralentissement de la croissance en hauteur observé chez les plantules. Cette croissance rapide de la plantule est un atout pour la régénération des ligneux en milieu aride. En effet, elle facilite la production rapide des plantules et leur adaptation rapide aux fluctuations climatiques et anthropiques [12].

Les performances germinatives des semences sont fonction des espèces, des traitements et des substrats. Des tests de germination ont été effectués sur des semences pré-traitées de 0 . insignis. Les résultats obtenus ont montré l'impact du prétraitement et du substrat sur la germination. La germination des semences témoins et prétraitées, est un atout pour la régénération sexuée de cette espèce. Les essais effectués en pots ont révélé que les semences sont aptes aux semis directs en champs et aux essais de plantation. L'étude apporte ainsi une solution en faveur de la culture des espèces locales notamment médicinales. 


\section{REMERCIEMENTS}

Nos remerciements vont Fonds National pour l'Education et la Recherche (FONER) du Burkina Faso, aux techniciens du Centre National de Semences Forestières (CNSF) de Ouagadougou et aux personnes morales pour leur assistance technique et leur soutien financier.

\section{REFERENCES BIBLIOGRAPHIQUES}

1. Ministère de la Santé, 2000. Document d'analyse de la situation nationale. 120p.

2. Nacoulma-Ouédraogo O.G., 1996. Plantes médicinales et pratiques médicales traditionnelles au Burkina Faso : cas du plateau central, Thèse, Faculté des Sciences et Techniques, Université de Ouagadougou, Burkina Faso, tome 1 et 2, 605p.

3. Bognounou O., Belem M. et Ilboudo A., 2000. Les plantes médicinales en péril au Burkina Faso. Communication orale à la 4ème édition du FIRST. Ouagadougou du 3 au 8 avril, 16p.

4. Zerbo P., Millogo-Rasolodimby J., NacoulmaOuédraogo O.G. et Van Damme P., 2007. Contribution à la connaissance des plantes médicinales utilisées dans les soins infantiles en pays San, au Burkina Faso. Int. J. Biol. Sci.1(3) : 262-274.

5. Bélem B. et Nana-Sanon P., 2009. Plantes médicinales utilisées pour le soin des enfants dans la ville de Ouagadougou, Burkina Faso, Afrique de l'ouest. Le Flamboyant 65 : 9-12p.

6. Lambert J.D.H., 2003. Vente et commercialisation des plantes médicinales à Ouagadougou et à Bobo-Dioulasso. Evaluation socio-économique, Canadian Trust Funds, 45p.

7. IPGRI, 2001. Programme de ressources génétiques forestières en Afrique au sud du Sahara (Programme SAFORGEN) Réseau Espèces ligneuses médicinales. Compte rendu de la première réunion du réseau 15 17 décembre 1999. Cotonou, Bénin. 131 p

8. OMS, 2002. Stratégie de l'OMS pour la médecine traditionnelle (2002 -2005). Genève. $78 p$.

9. Bationo B.A., Karim S., Bellefontaine R., Saadou M., Guinko S., Ichaou A. et Bouhari A., 2005. Le marcottage terrestre : une technique économique pour la régénération de certains ligneux tropicaux. Sécheresse 16 (4) : 309 - 311.

10. Bellefontaine R., Sabir M., Kokou K., Guinko S., Saadou M., Ichaou A., Hatem C., Bationo
B. A., Dourma M. et Karim S. 2005. Argumentaire pour l'étude et l'utilisation des marcottes et drageons dans les pays à faibles couverts ligneux. Sécheresse 16 (4): 312-314.

11. Thiombiano A., Wittig R. et Guinko S., 2003. Conditions de la multiplication sexuée chez les Combretaceae du Burkina Faso. Rev.Ecol. (Terre Vie) 58 : 361-379.

12. Ouédraogo A., Thiombiano A., Hann-Hadjiali K. et Guinko S., 2006. Régénération sexuée de Boswelia dalzielii Hutch., un arbre médicinal de grande valeur au Burkina Faso. Bois et Forêts des Tropiques 289 : 41-48.

13. Yélémou $\quad$ B., Yaméogo G., MillogoRasolodimby J. et Hien V., 2007. Germination sexuée et dynamique de développement de Piliostigma reticulatum (D.C.) Hochst., une espèce agroforestière du Burkina Faso. Sécheresse 18 (3) : 185-192.

14. Bélem B., Boussim J.I., Bellefontaine R. et Guinko S., 2008. Stimulation du drageonnage de Bombax costatum par blessure des racines au Burkina Faso. Bois et Forêts des Tropiques 295 (1) : 71-79.

15. Adjanohoun E.J., Adjakidjé V., Aké Assi L., Ahyi A.M.R., Akoegninou A., Almeida J., Apovo F., Boukef K., Chadaré M., Cusset G., Dramane K., Eymé J., Gassista J.-N., Gbaguidi N., Goudoté E., Guinko S., Houngnon P., Keita A., Kiniffo H.V., KonéBamba D., Musampa A., Saadou M., Sodogandji T., Souza De S., Tchabi A., Zinsou Dossa C. et Zohoun T., 1989. Médecine traditionnelle et pharmacopée Contribution aux études ethnobotaniques et floristiques en République Populaire du Bénin. ACCT, Paris : 185-199.

16. Mathabe M.C., Nikolova R.V., Lall N. et Nyazema N.Z., 2006. Antibacterial activities of medicinal plants used for the treatment of diarrhoea in Limpopo Province, South Africa. Journal of Ethnopharmacology 105 : 286-293.

17. Asase A., Oteng-Yeboah A.A., Odamtten G.T. et Simmonds M.S.J., 2005. Ethnobotanical study of some Ghanaian anti-malarial plants. Journal of Ethnopharmacology 99 : 273-279.

18. Runyoro D.K.B., Ngassapa O.D., Matee M.I.N., Joseph C.C. et Moshi M.J., 2006. Medicinal plants used by Tanzanian traditional healers in the management of Candida infections. Journal of Ethnopharmacology 106: 158-165. 
19. Fontes J. et Guinko S., 1995. Carte de la végétation et de l'occupation du sol du Burkina Faso. Note explicative. Ministère de la coopération française, projet Campus, Toulouse. 68p.

20. Arbonnier M., 2000. Arbres, arbustes et lianes des zones sèches d'Afrique de l'ouest. CIRAD, Paris, 541p.

21. Sawadogo I., 2005. Essai de la production de Securidaca longepedunculata Fresn., une espèce médicinale. Rapport de fin de stage pour le Diplôme des Préposés des Eaux et Forêts, ENEF, 37p.

22. Dorinta M., 2006. Essai de la production en pépinière de Ximenia americana L. Rapport de fin de stage pour le Diplôme des Contrôleurs des Eaux et Forêts, ENEF, 49p.

23. Néya O., 2006. Conservation of Tree Seeds from tropical Dry-lands. PhD thesis, Wageningen University, The Netherlands. 159p.

24. Yamégo W.M., 2009. Diversité des champignons endomycorhiziens et des bactéries fixatrices d'azotes associées aux niébé (Vigna unguiculata (L.) Walp.) dans différentes zones climatiques du Burkina Faso. Mémoire de fin de cycle d'ingénieur agronome. IDR, UPB, 73p. 